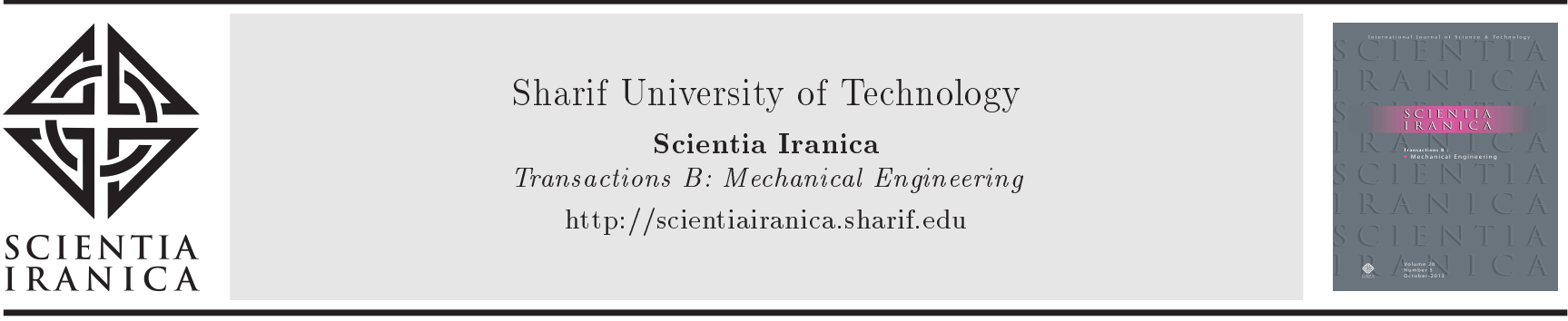

\title{
Double diffusive natural convection in a partially layered cavity with inner solid conductive body
}

\author{
M.A. Ismael* and H.S. Ghalib \\ Department of Mechanical Engineering, College of Engineering, University of Basrah, Basrah, Iraq.
}

Received 3 February 2017; received in revised form 26 May 2017; accepted 28 August 2017

\section{KEYWORDS}

Double-diffusive;

Cavity;

Porous medium;

Inner body;

Finite difference.

\begin{abstract}
This paper investigates the double-diffusive natural convection in a partially layered square cavity. The cavity is composed of a porous layer on the left and a fluid layer on the right. A conductive solid body is included in the cavity to control the natural convection. The left wall is kept at a constant high temperature and adjusted with high concentration, while the right wall is kept at a low temperature and low concentration. The horizontal walls are thermally insulated. The 2-dimensional governing equations have been solved using the upwind scheme finite difference method. The Parndtl number and buoyancy ratio are fixed at 6.26 and 1 , respectively. The problem has been governed by five parameters, namely Lewis number (Le $=1-50)$, Rayleigh number Ra $\left(10^{3}-10^{6}\right)$, Darcy number $\left(10^{-9}-1\right)$, aspect ratio of the body relative to the cavity $\mathrm{A}(0.3,0.5)$, and the position of the body. The results showed that locating the solid body close to the mid height of the left wall gives maximum convective heat transfer, while minimum heat transfer is associated when the solid body is located at the cavity center. It was also found that the Nusselt and Sherwood numbers behave in contradiction with the location of the solid body.

(C) 2018 Sharif University of Technology. All rights reserved.
\end{abstract}

\section{Introduction}

Heat and mass transfer processes concur in many technical applications. Specifically, mass transfer refers to the relative motion of species in a mixture due to concentration gradients. This topic subsumes the applications of geothermal reservoirs, extraction of petroleum, home humidifiers, chemical catalytic reactors, prevention of subsoil water pollution, nuclear reactors, underground diffusion of nuclear wastes and other contaminants, and porous material regenerative heat exchangers [1]. Due to their instrumental significance and relevance in engineering and industry, researchers have extensively studied heat and mass

*. Corresponding author.

E-mail addresses: muneerismael@yahoo.com (M.A. Ismael); muneer.ismael@uobasrah.edu.iq (H.S. Ghalib)

doi: $10.24200 /$ sci. 2017.4349 transfer (double-diffusive) in enclosed cavities composed of a porous layer confined or superposed by a clear fluid. Baytas et al. [2] investigated the double diffusive natural convection between a saturated porous layer and an overlying fluid layer in an enclosure using the non-Darcy flow model. Hirata et al. [3] studied a linear stability analysis of a thermosolutal natural convection in superposed fluid and porous layers. Islam et al. [4] investigated a double diffusive natural convection in a two-dimensional brine saturated porous medium, subjected to vertical concentration and temperature gradients. Hadidi et al. [5] analyzed numerically the heat and mass transfer generated in a partially horizontal porous enclosure. Their results showed that the presence of a porous layer has strong effects on the heat and mass transfer and the modification of the flow structure. Mharzi et al. [6] studied the natural thermosolutal convection in an elongated enclosure of horizontal axis, partitioned by a vertical porous layer. Their results showed the heat transfer affected by the 
thermal conductivity ratio, while the solutal transport is essentially sensitive to the solutal diffusivity ratio. Bennacer et al. [7] analyzed the double diffusive natural convection in an enclosure fitted with two symmetrical porous layers confining a fluid layer. Gobin et al. [8] investigated a natural convection driven by combined thermal and solutal buoyancy forces in a binary fluid. They confirmed that the presence of the porous layer has strong influence on the modification of the flow structure and consequent modifications of the heat transfer. Outaleb et al. [9] studied the double diffusive convection in a partially porous cavity with partially permeable walls under the combined buoyancy effects of thermal and mass diffusions. Hadidi et al. [10] investigated the double diffusive natural convection heat and mass transfer in a tilted porous cavity. Hadidi and Bennacer [11] extended the problem of [10] to twoand three-dimensional flows.

However, many studies have been published to passively enhance or attenuate the natural convection in enclosures by inserting conductive or adiabatic solids thermally. House et al. [12] performed a natural convection study in a differentially heated square enclosure containing a conductive square body. Ha and Jung [13] conducted a numerical study on three-dimensional heat transfer and flow characteristics of natural convection in a vertical cubic enclosure. They showed that the presence of a cubic conducting body in a cubic enclosure results in a larger variation of the local Nusselt number at hot and cold walls in $z$ direction, compared to cases without a cubic conducting body. Lee and $\mathrm{Ha}$ [14] investigated natural convection in a horizontal layer of fluid with an interior conductive body, heated from below and cold from above walls. Braga and de Lemos [15] presented numerical solutions for steady laminar and turbulent natural convection within a square cavity filled by a fixed amount of conducting solid material using a continuum model. Both continuum and porous continuum models presented higher values for the Nusselt number when turbulence was considered. In another study, Braga and de Lemos [16] investigated heat transfer across a square cavity partially filled with a fixed amount of a conductive solid body shaped with two different types of geometry. Merrikh and Lage [17] investigated the natural convection within a differentially heated heterogeneous square enclosure consisting of several disconnected conductive solid blocks within a saturated fluid. Das and Reddy [18] investigated the natural convection flow in a square enclosure with a centered internal conducting square block with an inclination angle. Zhao et al. [19] addressed the conjugate heat transfer in enclosures encircled with finite walls or with centered solid body.

Recently, Sheremet et al. [20] studied the problem of entropy generation and natural convection of nanofluid within a differentially heated cavity with a centered hot square block and corner cooler. They predicted that an increase in the centered solid block size leads to the formation of several convective cells of low intensity in a nanofluid gap between top cold wall and top surface of the heater. Sivaraj and Sheremet [21] studied the MHD natural convection in an inclined porous cavity with a heat conductive solid body under the influence of magnetic field applied to different orientations. They demonstrated that isotherms move away from the conducting body to increase the cavity angle, whereas they cluster toward the solid body in the presence of magnetic field. Chamkha et al. [22] performed a numerical simulation of mixed convection in a cavity that consists of a fluid and a superposed porous medium with an adiabatic cylinder rotating about the center of the cavity. They reported a linear increase of the average heat transfer with the angular rotational speed of the cylinder, and the increasing rate becomes higher as the cylinder size increases. Selimefendigil et al. [23] developed the work described in [22] by including $\mathrm{CuO}$ nanoparticles in the cavity with different vertical locations of the adiabatic rotating cylinder. They found that the local and average Nusselt number enhances when the cylinder approaches the upper wall of the cavity.

With respect to the above literature survey, it can be concluded that the double diffusive natural convection in a vertically partitioned-porous cavity with inner fixed conductive body has not been investigated yet. Hence, the goal of this paper is the numerical analysis of natural convection heat and mass transfer in such a cavity.

\section{Mathematical modelling}

The current problem is shown schematically in Figure 1 . It is a square cavity with side length $H$. A vertical water-saturated porous layer of thickness, $W_{p}$, is localized on the left side of the cavity, while pure water fills the remainder of the cavity. A conductive square body is included inside the cavity. The left wall of the cavity is heated isothermally (at $T_{h}$ ) and kept at high precise concentration, $c_{h}$. The right wall is cooled isothermally (at $T_{c}$ ) and kept at low precise concentration, $c_{L}$. The assumptions used with the proposed model are presented in the following. The flow is two-dimensional, incompressible, laminar, and steady state one. The porous medium is considered to be uniform with fixed pores between solid matrices. In addition, the fluid filling pores and solid matrix are assumed to be in thermal equilibrium. The physical properties are taken constant except the density where it obeys the Boussinesq's approximation. The energy dissipation and radiation of surface are negligible. Assuming that the convection within the porous layer 


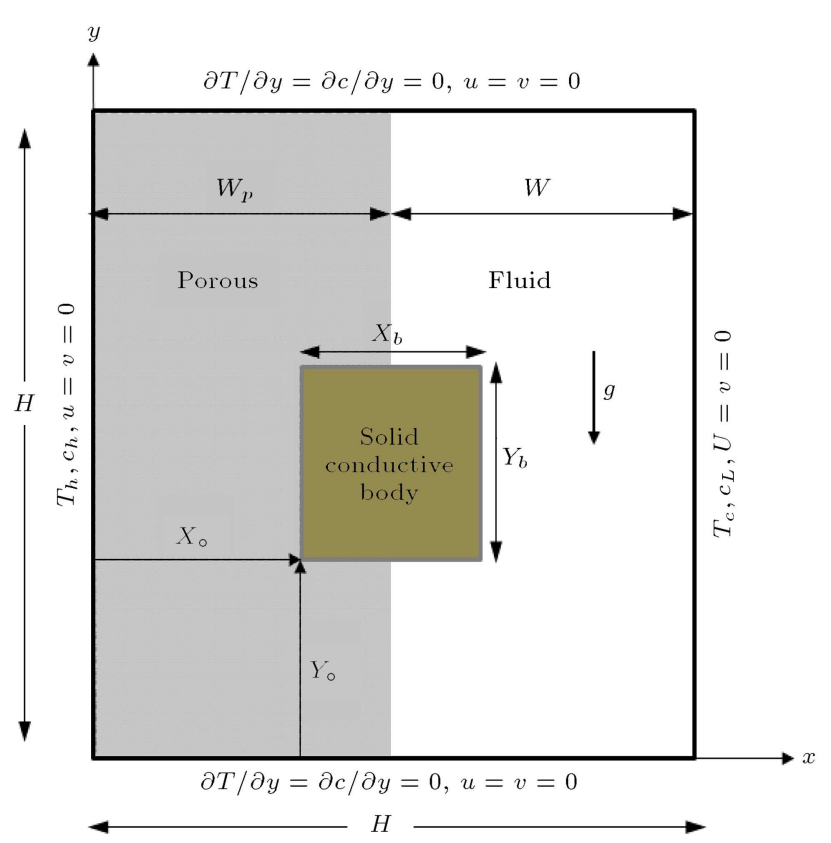

Figure 1. Physical domain, boundary conditions, and coordinate system.

occurs according to Darcy-Brinkman model and by invoking the stream function-vorticity formulation [24], dimensionless governing equations are as follows:

For porous layer:

$$
\begin{gathered}
\frac{\partial^{2} \Psi_{p}}{\partial X^{2}}+\frac{\partial^{2} \Psi_{p}}{\partial Y^{2}}=-\Omega_{p} \\
\frac{\partial \Psi_{P}}{\partial Y} \frac{\partial \Omega_{p}}{\partial X}-\frac{\partial \Psi_{P}}{\partial X} \frac{\partial \Omega_{P}}{\partial Y}=\varepsilon \operatorname{Pr}\left[\frac{\partial^{2} \Omega_{p}}{\partial X^{2}}+\frac{\partial^{2} \Omega_{p}}{\partial Y^{2}}\right] \\
\quad-\frac{\operatorname{Pr}}{\operatorname{Da}} \Omega_{p}+\varepsilon^{2} \operatorname{RaPr} \frac{\partial \theta_{p}}{\partial X}+\varepsilon^{2} \operatorname{RaPr} N \frac{\partial C_{p}}{\partial X} \\
\frac{\partial \Psi_{p}}{\partial Y} \frac{\partial \theta_{p}}{\partial X}-\frac{\partial \Psi_{p}}{\partial X} \frac{\partial \theta_{P}}{\partial Y}=\frac{\alpha_{\text {eff }}}{\alpha_{f}}\left[\frac{\partial^{2} \theta_{P}}{\partial X^{2}}+\frac{\partial^{2} \theta_{p}}{\partial Y^{2}}\right] \\
\frac{\partial \Psi_{p}}{\partial Y} \frac{\partial C_{p}}{\partial X}-\frac{\partial \Psi_{p}}{\partial X} \frac{\partial C_{P}}{\partial Y}=\frac{D_{\text {eff }}}{D_{f}} \frac{1}{L_{e}}\left[\frac{\partial^{2} C_{P}}{\partial X^{2}}+\frac{\partial^{2} C_{p}}{\partial Y^{2}}\right] .
\end{gathered}
$$

For fluid:

$$
\begin{aligned}
& \frac{\partial^{2} \Psi_{f}}{\partial X^{2}}+\frac{\partial^{2} \Psi_{f}}{\partial Y^{2}}=-\Omega_{f} \\
& \frac{\partial \Psi_{f}}{\partial Y} \frac{\partial \Omega_{f}}{\partial X}-\frac{\partial \Psi_{f}}{\partial X} \frac{\partial \Omega_{f}}{\partial Y}=\operatorname{Pr}\left[\frac{\partial^{2} \Omega_{f}}{\partial X^{2}}+\frac{\partial^{2} \Omega_{f}}{\partial Y^{2}}\right] \\
& \quad+\operatorname{RaPr} \frac{\partial \theta_{f}}{\partial X}+\operatorname{RaPr} N \frac{\partial C_{f}}{\partial X},
\end{aligned}
$$

$$
\begin{aligned}
& \frac{\partial \Psi_{f}}{\partial Y} \frac{\partial \theta_{f}}{\partial X}-\frac{\partial \Psi_{f}}{\partial X} \frac{\partial \theta_{f}}{\partial Y}=\left[\frac{\partial^{2} \theta_{f}}{\partial X^{2}}+\frac{\partial^{2} \theta_{f}}{\partial Y^{2}}\right] \\
& \frac{\partial \Psi_{f}}{\partial Y} \frac{\partial C_{f}}{\partial X}-\frac{\partial \Psi_{f}}{\partial X} \frac{\partial C_{f}}{\partial Y}=\frac{1}{L_{e}}\left[\frac{\partial^{2} C_{f}}{\partial X^{2}}+\frac{\partial^{2} C_{f}}{\partial Y^{2}}\right] .
\end{aligned}
$$

For a conductive body:

$$
\frac{\partial^{2} \theta_{b}}{\partial X^{2}}+\frac{\partial^{2} \theta_{b}}{\partial Y^{2}}=0
$$

where Prandtl number $(\mathrm{Pr})$ is equal to $\frac{v_{f}}{\alpha_{f}}$, Thermal Rayleigh number (Ra) is equal to $\frac{g \beta_{T, f} \Delta T H^{3}}{\alpha_{f} v_{f}}$, Darcy number (Da) is equal to $\frac{K}{H^{2}}$, Lewis number (Le) is equal to $\frac{\alpha_{f}}{D_{f}}$ and Buoyancy ratio is $N=\frac{\beta_{C} \Delta C}{\beta_{T} \Delta T}$.

The boundary conditions are:

1. On the left wall, $X=0$ :

$$
\Psi=0, \theta=1, C=1, \Omega=-\left(\frac{\partial^{2} \Psi}{\partial X^{2}}\right),
$$

2. On the right wall, $X=1$ :

$$
\Psi=0, \theta=0, C=0, \Omega=-\left(\frac{\partial^{2} \Psi}{\partial X^{2}}\right),
$$

3. On the bottom and top wall, $Y=0,1$ :

$$
\begin{aligned}
& \Psi=0, \partial \theta / \partial Y=0, \partial C / \partial Y=0, \\
& \Omega=-\left(\frac{\partial^{2} \Psi}{\partial Y^{2}}\right) .
\end{aligned}
$$

The solution at the interface is derived by considering the continuity of the tangential and normal velocities, temperature, mass and heat flux across the interface. One of the advantages of Darcy-Brinkman model is that the dynamic viscosities are taken to be the same in both layers $\left(\mu_{\mathrm{eff}}=\mu_{f}\right)$ [25]. Hence, the porous-fluid interface conditions can be written as follows:

$$
\theta_{P}=\theta_{f}, \quad \frac{\partial \theta_{f}}{\partial X}=\operatorname{Kr} \frac{\partial \theta_{p}}{\partial X},
$$

where $\mathrm{Kr}=\frac{k_{\text {eff }}}{k_{f}}$ is the thermal conductivity ratio.

$$
k_{\mathrm{eff}}=(1-\varepsilon) k_{s}+\varepsilon k_{f},
$$

where $k_{s}$ is the thermal conductivity of the solid matrix forming the porous interface:

$$
C_{P}=C_{f}, \quad \frac{\partial c_{f}}{\partial X}=\operatorname{Rd} \frac{\partial C_{p}}{\partial X},
$$

where $\mathrm{Rd}=\frac{D_{\text {eff }}}{D_{f}}$ is the mass diffusivity ratio.

$$
\begin{array}{ll}
\Psi_{p}=\Psi_{f}, & \frac{\partial \Psi_{f}}{\partial X}=\frac{\partial \Psi_{P}}{\partial X}, \\
\Omega_{P}=\Omega_{f}, & \frac{\partial \Omega_{f}}{\partial X}=\frac{\partial \Omega_{P}}{\partial X} .
\end{array}
$$

On the solid body (fluid side): 


$$
\begin{aligned}
& \theta_{b}=\theta_{f}, \quad \frac{\partial \theta_{f}}{\partial n}=\frac{k_{b}}{k_{f}} \frac{\partial \theta_{b}}{\partial n}, \\
& \frac{\partial C_{f}}{\partial n}=0 \\
& \Psi_{f}=0, \Omega_{f, 0}=-\frac{\partial^{2} \Psi_{f}}{\partial n^{2}} \\
& = \begin{cases}\frac{-8 \Psi_{0+1, j}+\Psi_{0+2, j}}{2 \Delta X^{2}} & \text { right groove } \\
\frac{-8 \Psi_{i, 0+2}+\Psi_{i, 0+2}}{2 \Delta Y^{2}} & \text { upper groove } \\
\frac{-8 \Psi_{i, 0-1}+\Psi_{i, 0-2}}{2 \Delta Y^{2}} & \text { lower groove }\end{cases}
\end{aligned}
$$

where $n$ is the normal vector, 0 represents the boundary node, 1 and 2 are the neighboring nodes to 0 , and $i$ and $j$ are the nodes series along $x$ and $y$ axes, respectively.

On the solid body (porous side):

$$
\begin{aligned}
& \theta_{b}=\theta_{p}, \quad \frac{\partial \theta_{p}}{\partial n}=\frac{k_{b}}{k_{\mathrm{eff}}} \frac{\partial \theta_{b}}{\partial n} \\
& \frac{\partial C_{p}}{\partial n}=0 \\
& \Psi_{p}=0, \Omega_{p, 0}=-\frac{\partial^{2} \Psi_{p}}{\partial n^{2}} \\
& = \begin{cases}\frac{-8 \Psi_{0-1, j}+\Psi_{0-2, j}}{2 \Delta X^{2}} & \text { Right groove } \\
\frac{-8 \Psi_{i, 0+1}+\Psi_{i, 0+2}}{2 \Delta Y^{2}} & \text { Upper groove } \\
\frac{-8 \Psi_{i, 0-1}+\Psi_{i, 0-2}}{2 \Delta Y^{2}} & \text { Lower groove }\end{cases}
\end{aligned}
$$

The local Nusselt number is defined as follows:

$$
\mathrm{Nu}=\frac{h H}{k}=\frac{-\frac{\partial T}{\partial x} H}{\left(T_{h}-T_{I}\right)}=-\frac{k_{\mathrm{eff}}}{\mathrm{k}_{f}} \frac{\partial \theta}{\partial X} .
$$

The average Nusselt number along the left wall is:

$$
\mathrm{Nu}_{\mathrm{av}}=\int_{0}^{1} \mathrm{Nu} d y
$$

The local Sherwood number is defined as follows:

$$
\mathrm{Sh}=-\frac{\partial C}{\partial X} \text {. }
$$

The average Sherwood number along the left wall is:

$$
\mathrm{Sh}_{\mathrm{av}}=\int_{0}^{1} \mathrm{Sh} d y \text {. }
$$

\section{Numerical implementation and validations}

\subsection{Numerical method}

In the numerical solution, the central finite difference method was adopted. Firstly, the physical domain was discretized to uniform $N x \times N y$ grids. Then, governing Eqs. (1) to (9) were solved using the Gauss-Seidel iteration procedure with the Successive Under-Relaxation (SUR) method. An upwind scheme procedure has been considered herein to attain the stability of a numerical solution with the convective and diffusive terms of the momentum and energy equations. The stream function, vorticity, mass and dimensionless temperature have been computed based on continuity, momentum, mass and energy equations, respectively. It should be mentioned that an in-house computer code (in FORTRAN) was built to obtain a numerical solution.

\subsection{Validation}

\subsubsection{Grid independency test}

An extensive mesh testing procedure was conducted to guarantee the achievement of a grid independent solution. In Figure 2(a), various mesh combinations were explored for:

$$
\begin{aligned}
& \text { Da }=10-3, \quad \mathrm{Ra}=10^{6}, \quad \mathrm{Le}=50, \quad N=1, \\
& \operatorname{Pr}=6.26, \quad W_{p}=0.5, \quad A=0.3, \quad \mathrm{Kr}=\frac{k_{b}}{k_{f}}=5 .
\end{aligned}
$$

The present numerical solution was tested for grid independence by calculating the average Nusselt number and Sherwood number on the heated vertical left wall. Based on the relevant criteria (solution accuracy and the time consumed by the processor), a grid size of $91 \times 91$ ensures the aforementioned criteria. This uniform grid distribution is shown in Figure 2(b). It is worth mentioning that the porous layer's thickness and solid body size were selected so that the interface boundaries could be localized on the discretized grid nodes.

\subsubsection{Comparisons with others}

The present numerical solution was further validated by comparing its results for $\mathrm{Ra}=10^{6}, N=10, \mathrm{Pr}=$ $10, W_{p}=0.1$, and aspect ratio $=2$ (height-to-width


Figure 2a. Grid independency test, $\mathrm{Ra}=10^{6}$, Le $=50$, $N=1, \operatorname{Pr}=6.26, W_{p}=0.5, \mathrm{Da}=10, \mathrm{~A}=0.5$, and $\mathrm{Kr}=5$ 


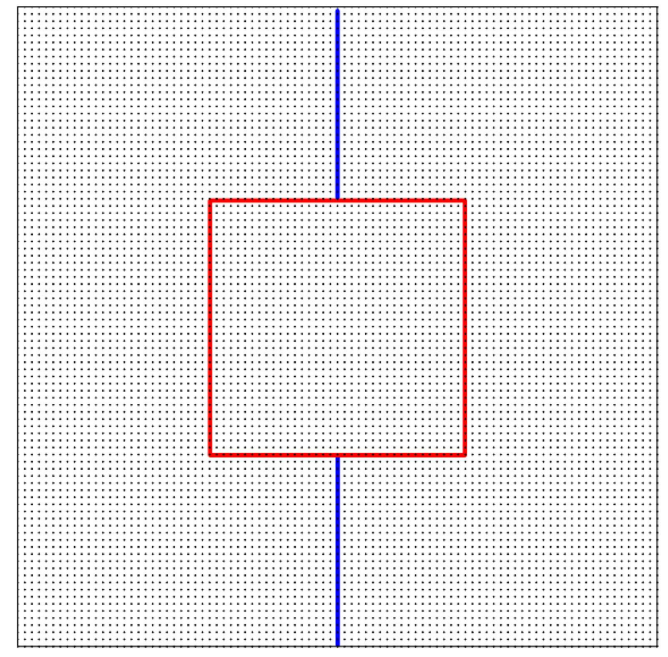

Figure 2b. Uniform grid distribution of $91 \times 91$.

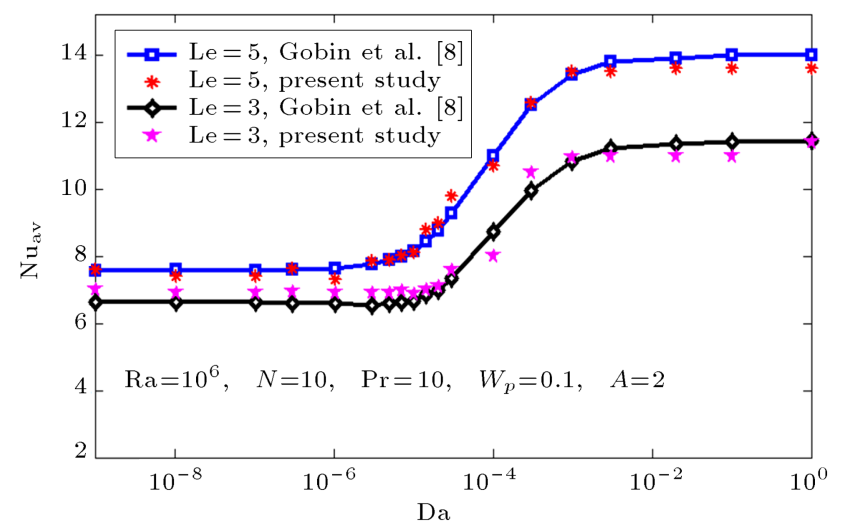

Figure 3. Comparison of average Nusselt number with Gobin et al. [8].

ratio of cavity) different other parameters with the numerical results of Gobin et al. [8] under the same thermal and concentration boundary conditions. The tests are presented as the variation of average Nusselt number with different Darcy and Lewis numbers. As shown in Figure 3, the present numerical solution is in good agreement with that of Gobin et al. [8]. In accordance with these two validations, the present code can be used to obtain the results with a high confidence level.

\section{Results and discussion}

The results of the present paper are illustrated graphically by contour maps of stream function, isotherms, isoconcentration, and the average Nusselt and Sherwood numbers. The parameters affecting the convection inside the present composite cavity include Raleigh number, Ra, Darcy number, Da, Lewis number, Le, bouncy ratio, $N$, conductivity ratio, $\mathrm{Kr}$, and the position of the inner conductive body. The ranges of selective parameters are as follows: $\mathrm{Ra}=10^{3}-10^{6}$, Le
$=1-50, \mathrm{Da}=10^{-9}-1, \mathrm{Kr}=(0.44,1$, and 5$)$, and five positions of the solid body. The other parameters are fixed at $N=1$, and $\operatorname{Pr}=6.26$.

Physically, Rayleigh number represents the ratio of the thermal buoyancy to viscous forces. Lewis number is the ratio of thermal diffusivity to mass diffusivity, and the buoyancy ratio reflects the solutal to thermal buoyancy.

The value of the porosity is taken to be $\varepsilon=$ 0.398 , which identifies glass beads of $3 \mathrm{~mm}$ diameter with a thermal conductivity of $0.845 \mathrm{~W} / \mathrm{m} . \mathrm{k}$ [25]. In accordance with the procedure followed by Juan et al. [26], mass diffusivity ratio $\mathrm{Rd}=\frac{D_{\text {eff }}}{D_{f}}$ is estimated in the current study to be 0.5322 .

\subsection{Influence of the Darcy number}

Darcy number represents the dimensionless permeability of the porous medium. The lesser the Darcy number is, the larger the resistance of the porous medium will be. Due to the low mass diffusivity ratio (0.5322) considered in this study, it is believed that the effect of Darcy number can be accompanied with the Rayleigh number. Therefore, the influence of Darcy number is investigated for more than one $\mathrm{Ra}$ value, as shown in Figures 4 and 5 . The other parameters are fixed at Le $=10, N=1, A=0.5$, and $\mathrm{Kr}=5$. Figure 4 shows the behavior of the three contour maps at Ra $=10^{4}$. When Da $=10^{-3}$ (Figure 4(a)), the flow is intensified out of the porous layer; however, the porous layer exhibits some flow. When Da number decreases to $10^{-5}$ (Figure $4(\mathrm{~b})$ ), the resistance exerted by the porous medium increases; thus, the flow is completely stagnant within the porous region, while the strength of the streamlines elsewhere decreases. This pattern becomes clear at $\mathrm{Da}=10^{-7}$ (Figure $4(\mathrm{c})$ ).

The isotherms, in general, manifest a contracting fashion in the middle of the cavity due to the pure conduction in the square solid body, accelerating the transverse heat transfer. However, when $\mathrm{Da}=10^{-3}$, a gradient in the isotherms exists within the porous layer, while this layer looks isothermal when Da decreases to $10^{-5}$ and $10^{-7}$. This refers to the restricted heat transfer there. The isoconcentration maps show that species' gradients can clearly be characterized only when $\mathrm{Da}=10^{-3}$ (Figure $4(\mathrm{a})$ ). When Da number decreases to $10^{-5}$ and $10^{-7}$, the restricted buoyancy convection becomes insufficient to motivate the species; thus, the isoconcentration lines look vertical with approximately equal potentials in the horizontal grooves (where "groove" is a horizontal or vertical path between the solid body surface and cavity wall). For a relatively higher Rayleigh number $\left(\mathrm{Ra}=10^{5}\right)$, as shown in Figure 5, streamlines are significantly strengthened due to the buoyancy effect. However, they reserve their behavior with Da number the same as that seen in $\mathrm{Ra}=$ $10^{4}$. Some perturbations occur with the isotherms, 




(a) $\mathrm{Da}=10^{-3}$

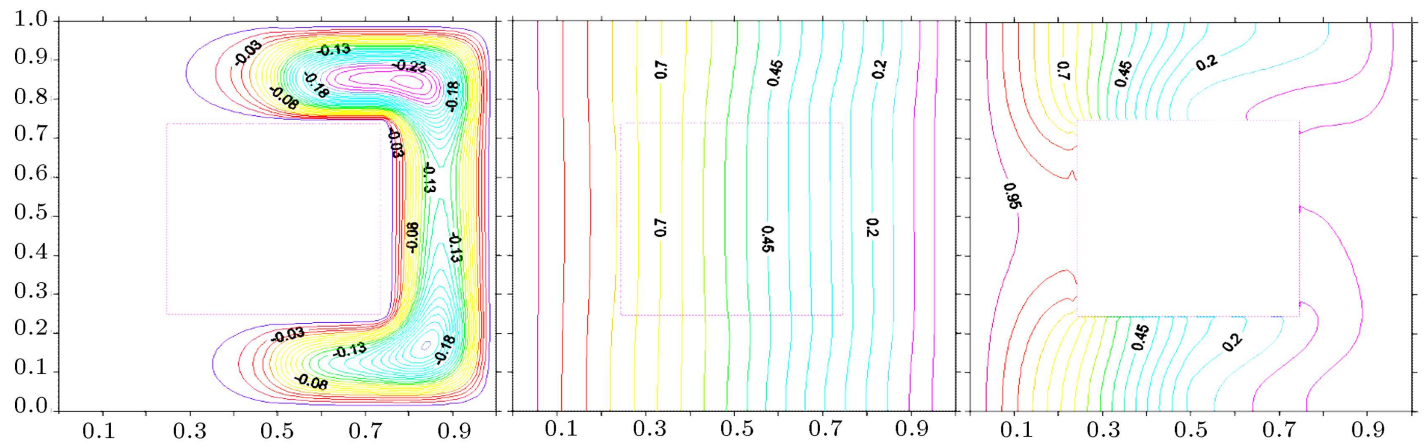

(b) $\mathrm{Da}=10^{-5}$

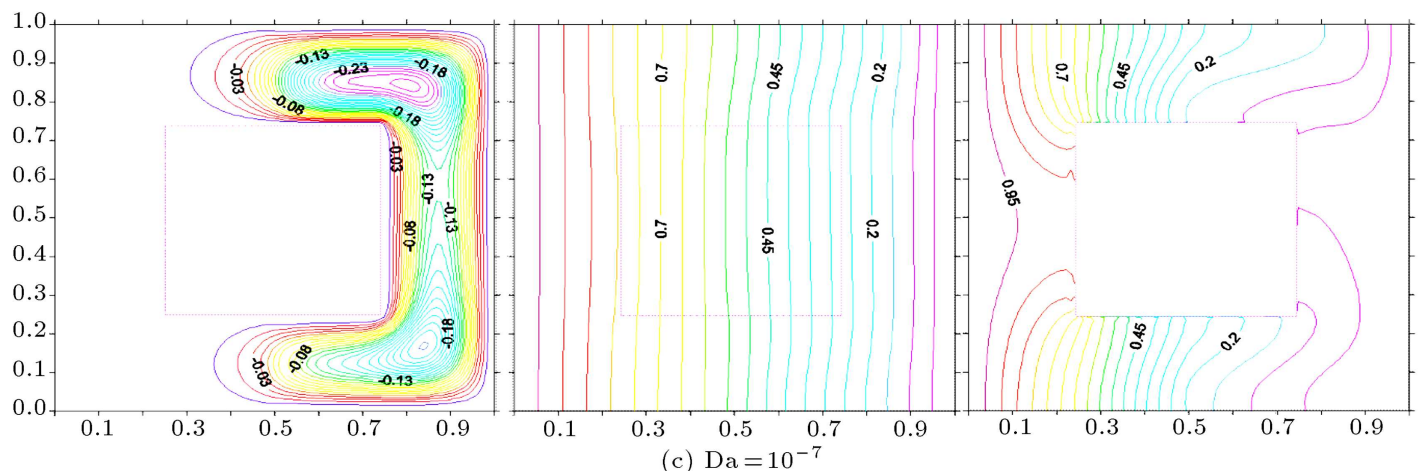

Figure 4. Streamlines, isotherms, and isoconcentrations for selected values of Darcy number; Le $=10, N=1, A=0.5, \mathrm{Kr}$ $=1$, and $\mathrm{Ra}=10^{4}$.

especially within the horizontal grooves. For Da $\geq$ $10^{-5}$, the isotherms' patterns become featureless. The isoconcentrations of $\mathrm{Da}=10^{-3}$ (Figure 5(a)) exhibit good mixing of species, where a remarkable gradient is seen within the porous layer (left groove) and upper groove, with thin solutal boundary layers there. By increasing resistance of the porous layer, i.e., decreasing Darcy number, the concentration gradient is noticeably affected (Figure 5(b) and (c)), where the right side of the cavity manifests no mass transfer, while some concentration gradients exist within the porous layer; this is due to low permeability and tortuous effects resulted from the porous layer.

The effects of Darcy number on the average Sherwood and Nusselt numbers are depicted in Figures 6 and 7 , respectively, for $\mathrm{Ra}=10^{5}$ and different Le numbers. These figures show that there is no significant influence of Darcy number on the mass transfer within the range of $\mathrm{Da} \leq 10^{-6}$, while the convective heat transfer is diminished within the range of $\mathrm{Da} \leq 10^{-5}$, which refers to the extremely low permeability of the porous layer, hence restricting the movement of natural convection and, consequently, the solutal convection. Generally, the concentrated mass transfer starts at $\mathrm{Da} \approx 10^{-6}$ and the thermal convection at $\mathrm{Da} \approx$ $10^{-5}$. Both thermal and solutal convections increase rapidly until $\mathrm{Da} \approx 10^{-2}$. Beyond this, double diffusion becomes irrespective of the further increase of the Darcy number. This can be attributed to the low permeability ( $\mathrm{Da} \leq 10^{-5}$ ) of the porous layer, which acts as a blockage to the movement of fluid and species. The effect of blockage diminishes when $\mathrm{Da} \geq 10^{-5}$, and the porous layer acts as a fluid layer beyond $\mathrm{Da}=10^{-2}$ [8]. These figures reveal the effect of Lewis number where as Le increases, thickness of the solutal boundary layers decreases; hence, the Sherwood number increases 

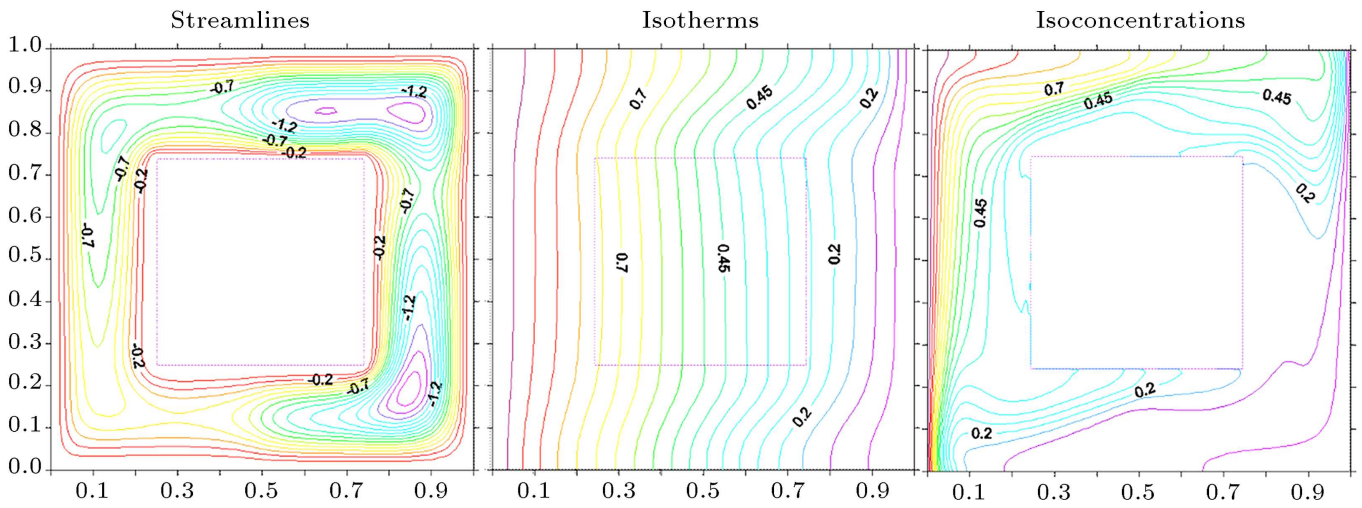

(a) $\mathrm{Da}=10^{-3}$

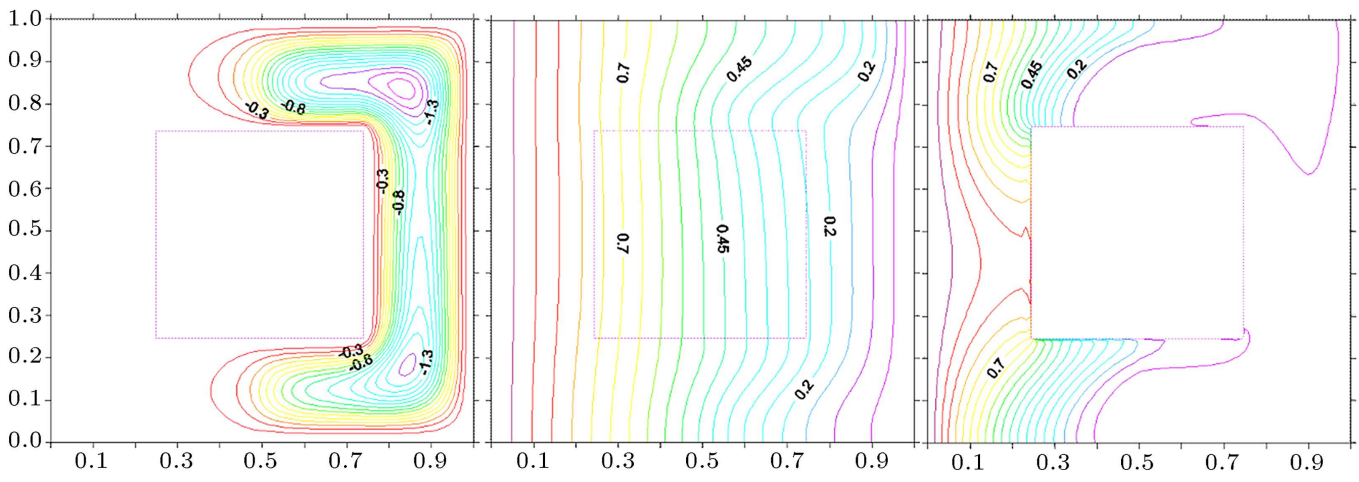

(b) $\mathrm{Da}=10^{-5}$



(c) $\mathrm{Da}=10^{-7}$

Figure 5. Streamlines, isotherms, and isoconcentrations for selected values of Darcy number, Le $=10, N=1, A=0.5, \mathrm{Kr}$ $=1$, and Rayleigh number $\mathrm{Ra}=10^{5}$.



Figure 6. Effects of Darcy number on Sherwood number for different Lewis numbers at $\mathrm{Ra}=10^{5}, N=1, A=0.5$, $W_{p}=0.5$, and $\mathrm{Kr}=1.0$



Figure 7. Effect of Darcy number on Nusselt number for different Lewis numbers at $\mathrm{Ra}=10^{5}, N=1, A=0.5$, $W_{p}=0.5$, and $\mathrm{Kr}=1$. 
uniformly. On the other hand, the thickness of the thermal boundary layer remains unchanged beyond Le $=10$.

\subsection{Influence of Lewis number}

Figure 8 shows the effect of Lewis number, Le, inspected by fixing other parameters/constants at Ra $=10^{4}, N=1$. As mentioned above, the Lewis number governs the ratio of thermal to mass diffusivity. Therefore, increasing Le can be interpreted either by the dominance of the thermal diffusivity, which restricts the convective heat transfer, or by diminishing the mass diffusivity with respect to thermal diffusivity. It is clear from Figure 8 that the streamlines at Le $=1$ are dense and strong throughout the cavity, such that, in the fluid layer (right groove), the stream function is stronger due to less flow resistance. However, when Le increases to 50 (Figure 8(c)), the flow intensity within the porous layer is notably reduced, characterized by less density streamlines. It is found that, at Le $=1$, the value of maximum absolute stream function is $|\psi|_{\text {max }}$ while, at $\mathrm{Le}=50$, it decreases to 0.24 . The isotherms appear to be unaffected by increasing Le number, which may be due to low buoyancy force $\left(\mathrm{Ra}=10^{4}\right)$. At Le $=1.0$, the equivalent thermal and mass diffusivities result in vertical iso concentration lines, especially in the upper and lower grooves. When Le increases to 30 and further to 50, thinner solutal boundary layers form close to the vertical cavity walls, while the dominant thermal diffusivity makes the iso concentration lines almost horizontal within the upper groove.

\subsection{Influence of Rayleigh number}

It is well known that the Rayleigh number judges the buoyancy force over the viscous force; however, it is studied herein to inspect its effect on the mass transfer. Figure 9 presents this effect for Le $=10$ and $N=1$. Patterns of the streamlines variations can be characterized by their intensity strengthened with Ra. It is found that the maximum absolute stream
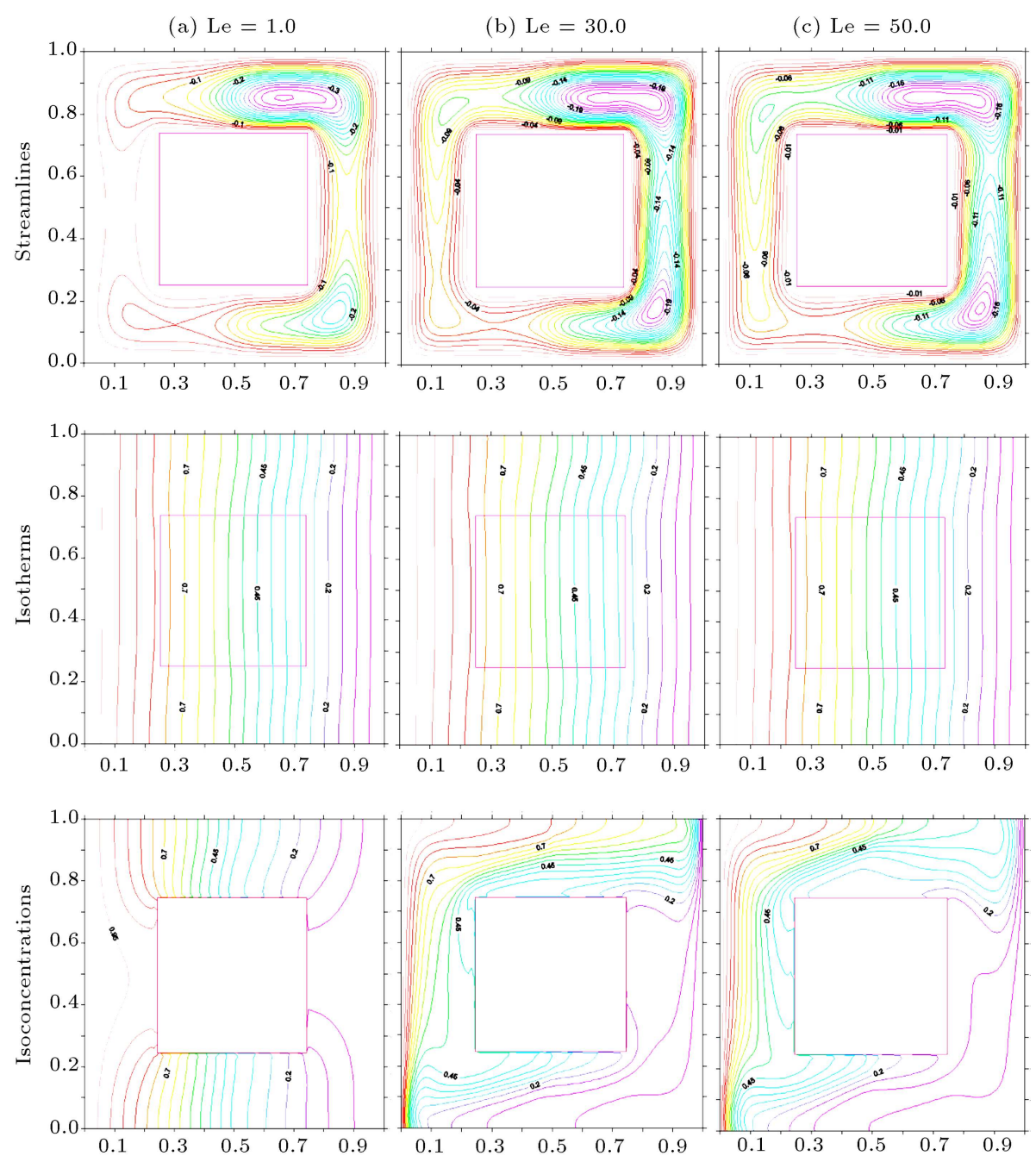

Figure 8. Streamlines, isotherms, and isoconcentrations contours for selected values of Lewis number, at Ra $=10^{4}$, $N=1, \mathrm{Da}=10^{-3}, A=0.5$, and $\mathrm{Kr}=1$. 

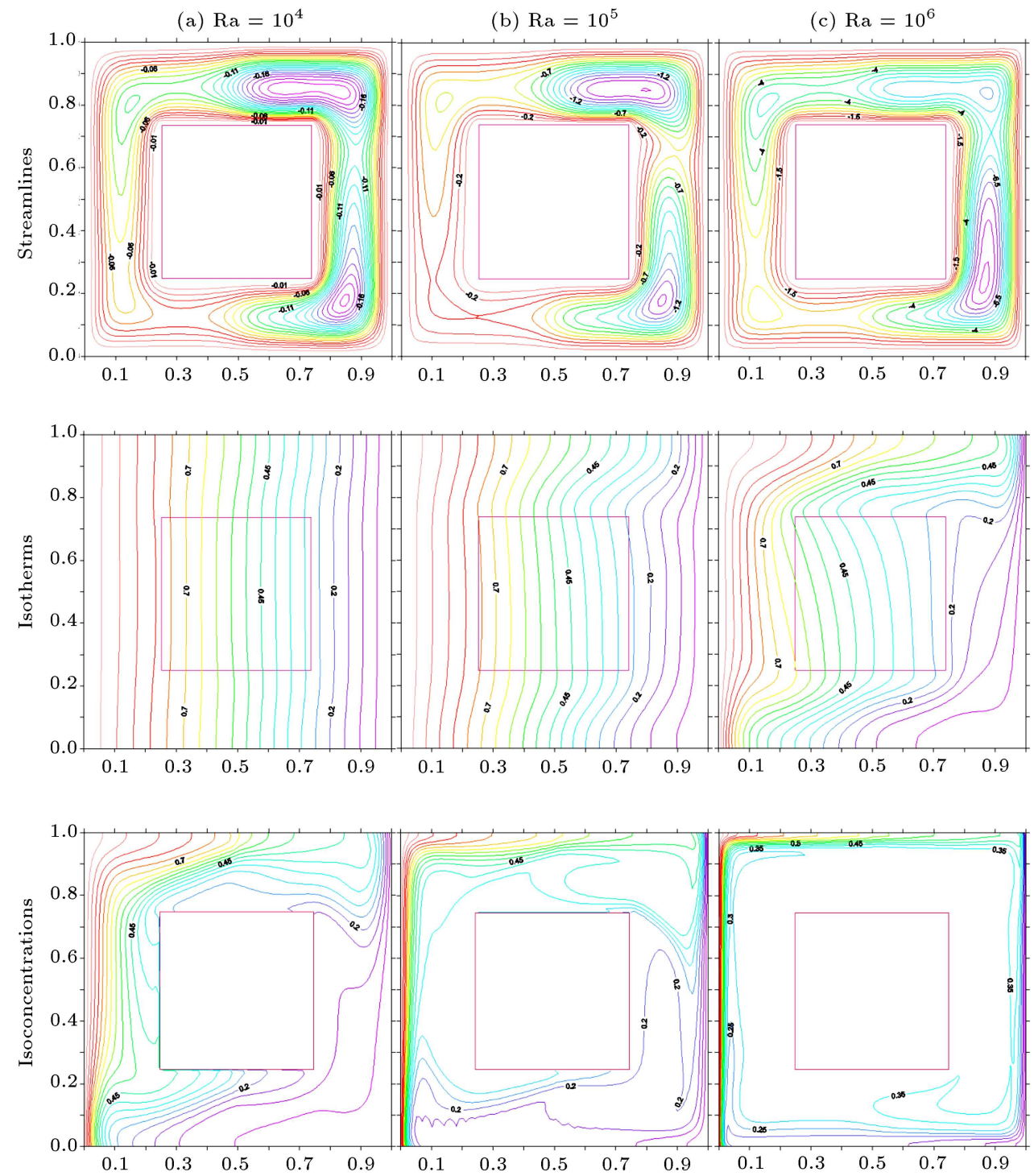

Figure 9. Streamlines, isotherms, and isoconcentrations contours for selected values of Rayleigh number, at Le $=1.0$, $N=1, \mathrm{Da}=10^{-3}, A=0.5$, and $\mathrm{Kr}=1$.

function $|\Psi|_{\max }$ is equal to $0.24,2.539$, and 12.103 when $\mathrm{Ra}$ increased from $10^{4}, 10^{5}$, to $10^{6}$, respectively. The isotherms are vertically distributed at $\mathrm{Ra}=10^{4}$ as an indication of the conduction dominance. At Ra $=10^{5}$, slight convective heat transfer takes place at the horizontal grooves. If $\mathrm{Ra}$ increases further to $10^{6}$, the existing relatively thin thermal boundary layer and the stratification behavior of the isotherms, especially in the horizontal grooves, imply the dominance of the convective heat transfer.

If the mechanism of the convective currents is strong, these currents can carry the species. This is clear in Figure 9 (lower row) where the isoconcentration lines become denser (thinner solutal boundary layer) close to the solid walls. Moreover, the concentration gradients settle close to the solid boundary, while the middle of the cavity is free of mass transfer.

\subsection{The influence of the thermal conductivity ratio}

Variations of the three contour maps have been examined for three different conductivity ratios, $\mathrm{Kr}=0.44$, 1.0 , and 5 (they correspond to epoxy-water, brickworkwater, and Granite-water, respectively [27]) and for two values of Rayleigh numbers, as shown in Figures 10 and 11. For $\mathrm{Ra}=10^{4}$, when $\mathrm{Kr}$ increases from 0.44 to 5 , the thermal resistance of the square body decreases accordingly. Thus, the enhanced conductive heat transfer augments the recirculation of fluid as shown clearly by the strengthening of the streamlines in the right upper and lower corners of the cavity. The gradients of the isotherms within the square body are high at low $\mathrm{Kr}$ and low at high $\mathrm{Kr}$ values; thus, the global behavior of the isotherms within the cavity is as follows: contracted at low $\mathrm{Kr}$, uniform at equivalent 

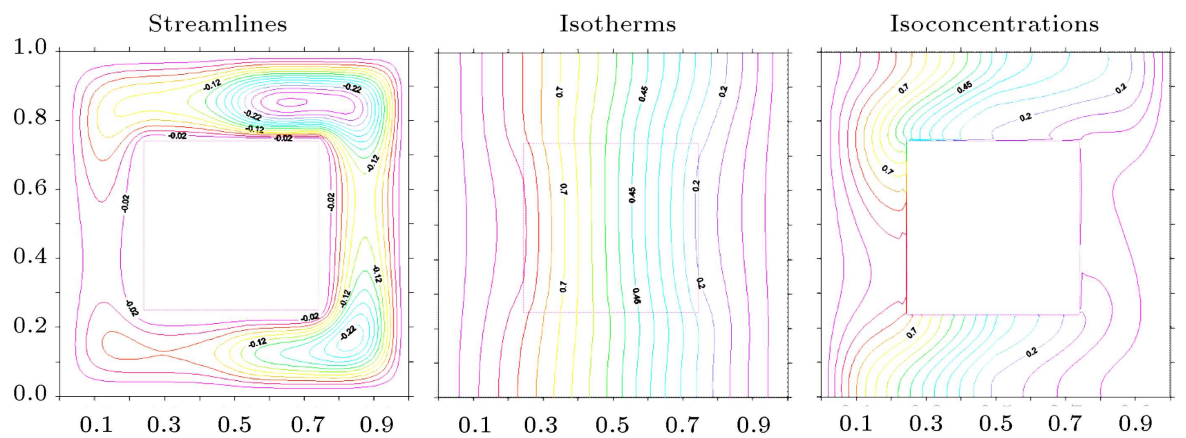

(a) $\mathrm{Kr}=0.44$


(b) $\mathrm{Kr}=1.00$


(c) $\mathrm{Kr}=5.00$

Figure 10. Streamlines, isotherms, and isoconcentrations for selected values of conductivity ratio; Le $=10, N=1$, $A=0.5$, and $\mathrm{Ra}=10^{4}$.

$\mathrm{Kr}(\mathrm{Kr}=1.0)$, and expanded at high $\mathrm{Kr}$, as shown in the middle row of Figure 10. The isoconcentration lines appear to be less affected by the conductivity ratio at $\mathrm{Ra}=10^{4}$ due to the unchanged convective heat transfer. However, increasing $\mathrm{Ra}$ number can affect the buoyancy force, which in turn strengthens the streamlines, stratifies the isotherms, and disturbs the isoconcentration lines. Hence, the behaviors of the remaining contours map of $\mathrm{Ra}=10^{5}$ (Figure 11) can be attributed accordingly.

Figures 12 and 13 clarify the effect of conductivity ratio, Kr, on Nusselt and Sherwood numbers. The higher the conductivity ratio is, the lower the thermal resistance of the solid body will be. This enables more heat transfer from the porous layer to the fluid layer across the solid body. Figures 12 and 13 depict the effect of $\mathrm{Kr}$ on a relatively moderate Rayleigh number $\left(\mathrm{Ra}=10^{5}\right)$. It is very clear from Figure 12 that the average Nusselt number increases with increasing Kr, which is due to the reduced thermal resistance of the solid body. Since the species do not penetrate the solid body, the role of $\mathrm{Kr}$ in the mass transfer (Sherwood number) will be indirect, that is, the mass transfer may be influenced by the thermal convection currents.

\subsection{The influence of the body location}

Let us imagine the present cavity free of the solid square body. The expected circulation of the fluid is a single $\mathrm{CW}$ vortex. This is due to a differentially heating boundary condition. Insertion of a solid body into a certain location can markedly alter the flow, heat, and mass lines. This category presents the effect of the body location on the fluid, thermal, and mass fields within the cavity. The other parameters are fixed at $\mathrm{Da}=10^{-3}, \mathrm{Le}=10, N=1, A=0.3, \mathrm{Kr}=5$, and $\mathrm{Ra}$ $=10^{5}$. Five body positions have been investigated by varying the body position horizontally and vertically as follows. 



(a) $\mathrm{Kr}=0.44$
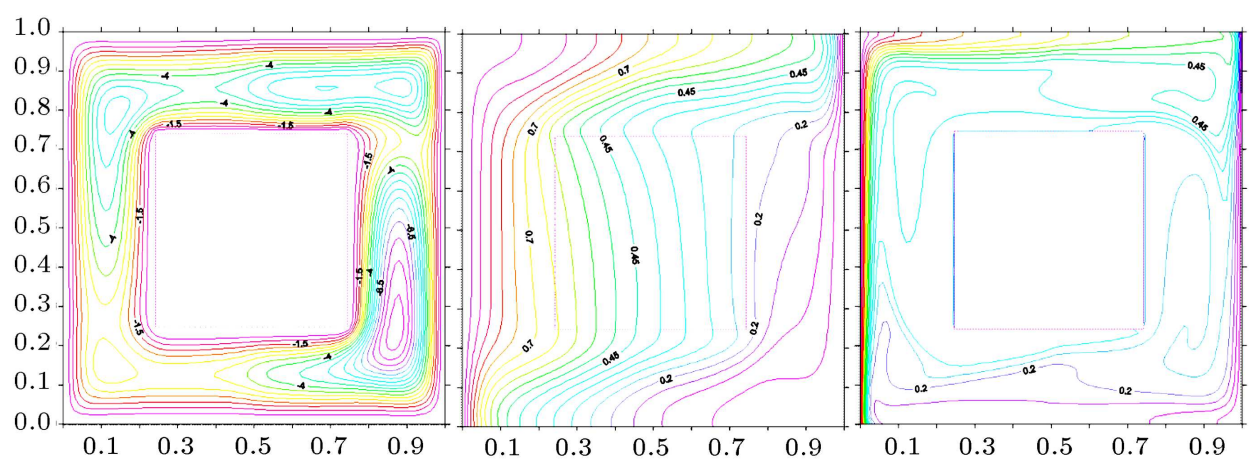

(b) $\mathrm{Kr}=1.00$

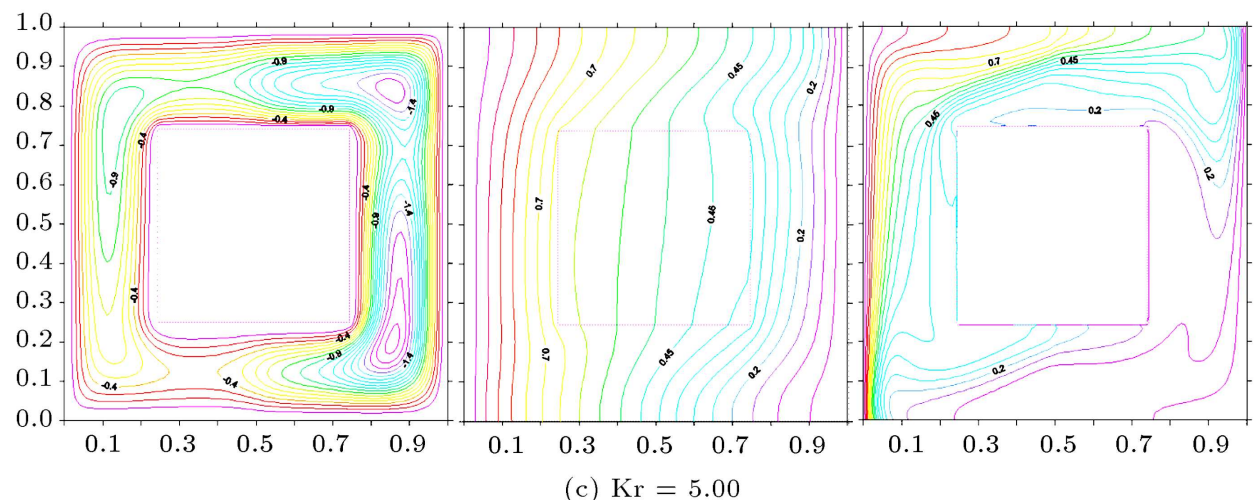

Figure 11. Streamlines, isotherms, and isoconcentrations for selected values of conductivity ratio; Le $=10, N=1$, $A=0.5$, and $\mathrm{Ra}=10^{5}$.

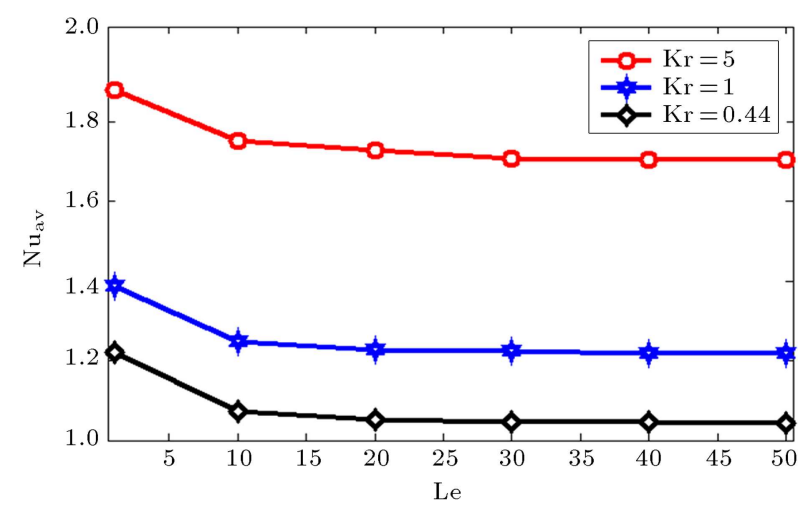

Figure 12. Effect of thermal conductivity ratio on the average Nusselt number for $\mathrm{Da}=10^{-3}, N=1, A=0.5$, and $\mathrm{Ra}=10^{5}$.

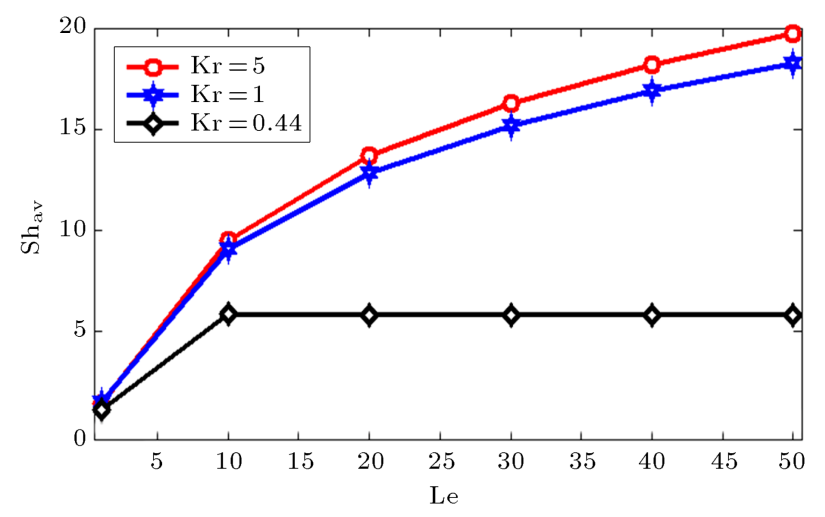

Figure 13. Effect of thermal conductivity ratio on the average Sherwood number for $\mathrm{Da}=10^{-3}, N=1, A=0.5$, and $\mathrm{Ra}=10^{5}$. 


\subsubsection{Various horizontal body locations}

The horizontal body locations were chosen such that the body could be close to the left hot wall within porous layer, $X_{o}=0.03$ (Case $1 \mathrm{H}$ ), in the middle of the cavity, concerted between the porous and fluid layers, $X_{o}=0.35($ Case $2 \mathrm{H})$, and close to the right cold wall within the fluid layer, $X_{o}=0.586$ (Case $3 \mathrm{H}$ ). Vertical positions are fixed at $Y_{o}=0.35$. In Case $1 \mathrm{H}$, the body restricts the flow, which is inherently weakened due to the tortuous path of the porous layer. Then, the flow circulates in the remainder of the cavity and concentrates in the fluid layer to form a single core vortex (Figure 14(a)). When the body shifts to the cavity center (Case $2 \mathrm{H}$ ), the flow initiates in the high resistance porous layer and circulates within the whole cavity, involving the solid body (Figure 14(b)). Due to the third horizontal location (Case $3 \mathrm{H}$ ), it is expected that the body location may exert extra retardation effect on the streamlines, where the flow in this region falls by gravity and moves freely (Figure 14(c)). Thus, Table 1 presents that, as expected, the flow strength decreases obviously with the horizontal locations for all Rayleigh numbers.

The body location of Case $1 \mathrm{H}$ gives permit to the isotherms to be arranged in a horizontal pattern in the fluid layer, enhancing the convective heat transfer. The existence of the body at the interface (Case $2 \mathrm{H}$ ) may act as a disturber to the heat transferred there. Therefore, the thermal boundary layers appear to be thicker. The disturber action of the body decreases as

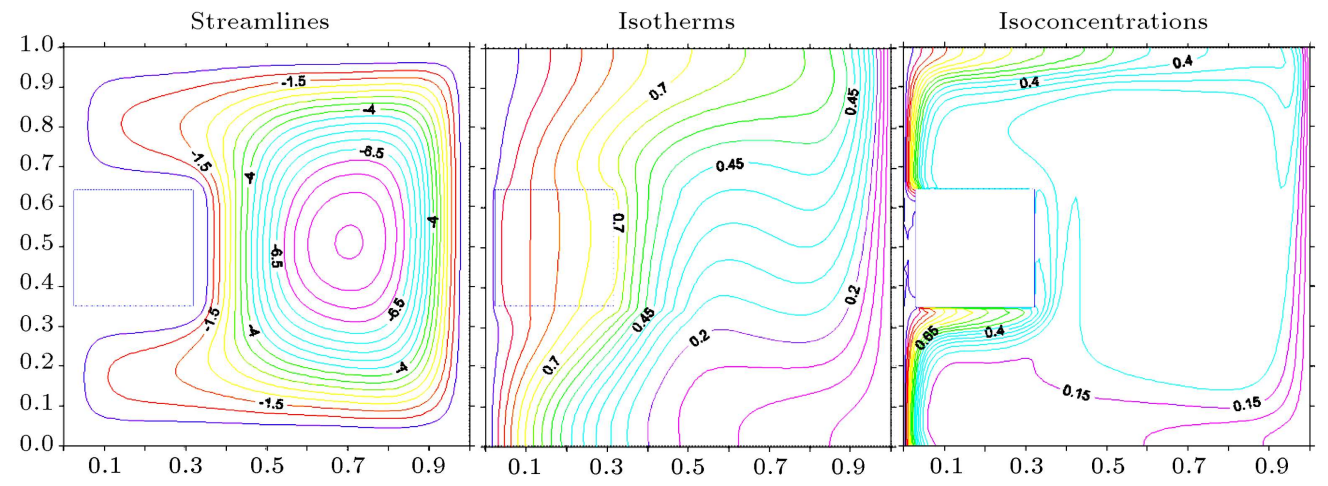

(a) Case $1 \mathrm{H}$

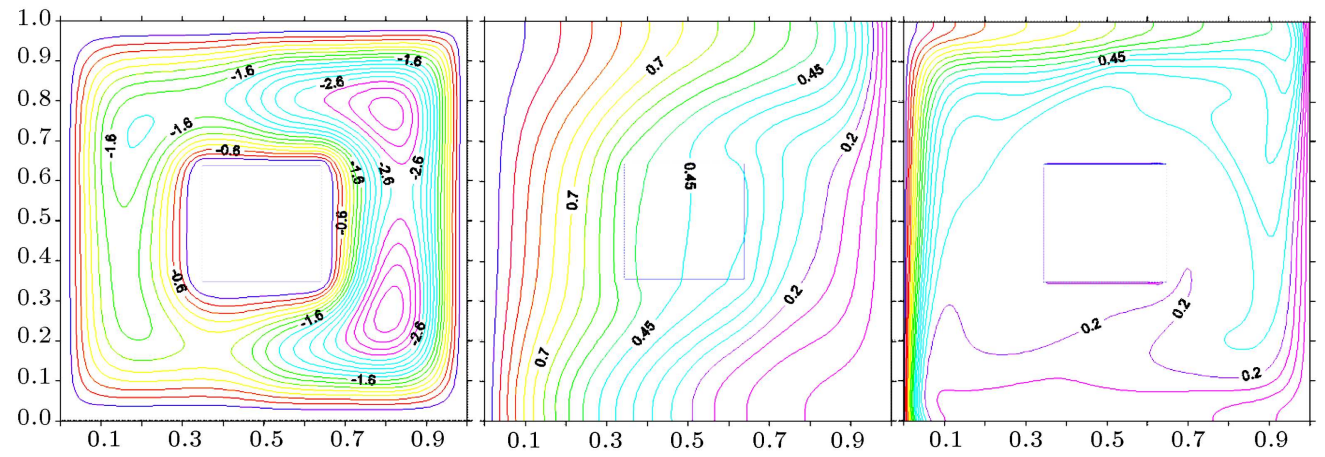

(b) Case $2 \mathrm{H}$

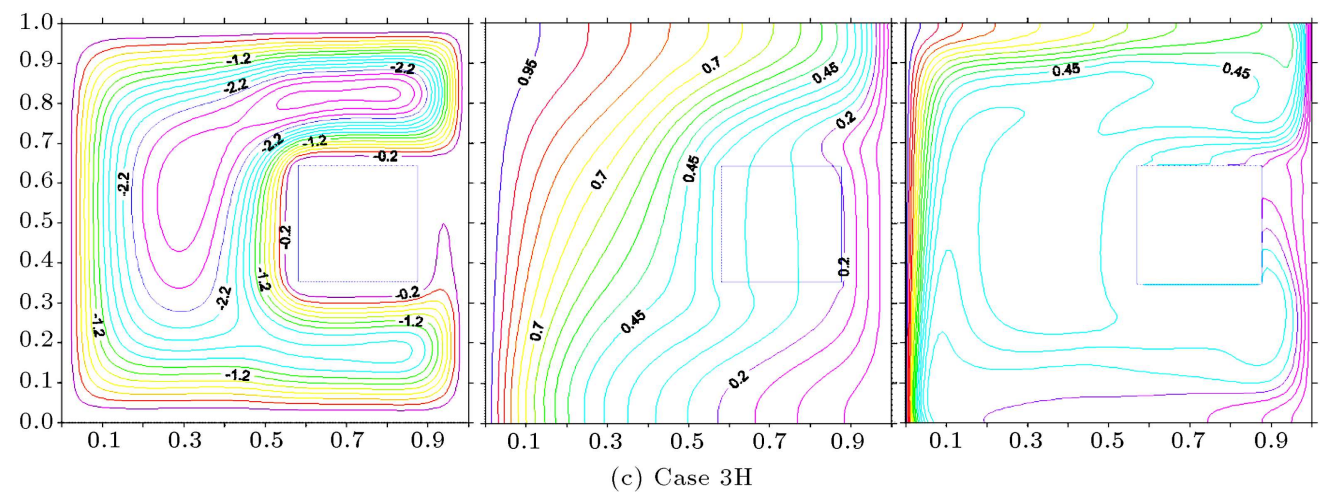

Figure 14. Effects of the horizontal body position on streamlines, isotherms, and isoconcentrations contours for Da $=$ $10^{-3}, \mathrm{Le}=10, N=1, A=0.3, \mathrm{Kr}=5$, and $\mathrm{Ra}=10^{5}$. 
Table 1. Minimum stream functions' values as a function of the horizontal body position and Rayleigh number for $\mathrm{Da}=10^{-3}, \mathrm{Le}=10$, and $\mathrm{Kr}=5$.

\begin{tabular}{cccc} 
Ra & Case 1H & Case 2H & Case 3H \\
\hline $10^{3}$ & $-2.80 \mathrm{E}-01$ & $-1.21 \mathrm{E}-01$ & $-7.44 \mathrm{E}-02$ \\
& & & \\
$10^{4}$ & -2.14557 & -0.7805 & $-5.38 \mathrm{E}-01$ \\
& & & \\
$10^{5}$ & -8.5644 & -3.53763 & -3.06811 \\
& & & \\
$10^{6}$ & -23.662 & -13.1729 & -14.2767 \\
\hline
\end{tabular}

it shifts to $3 \mathrm{H}$ position, where the streamlines reserve the thermal boundary layers, especially close to the upper part of the right wall (Figure 14(c)).

Because the species source is the left wall, the solutal boundary layer is noticeably destroyed when the body is located close to the left wall (Case $1 \mathrm{H})$, where there is insufficient thermal convection to carry the species. For other locations $(2 \mathrm{H}$ and $3 \mathrm{H})$, the species are carried freely to the remainder of the cavity. This is demonstrated by the reserved solutal boundary layers (Figure 14(b) and (c)).

The average Nusselt and Sherwood numbers are plotted in Figures 15 and 16, respectively, against Rayleigh number and for the three different horizontal locations of the solid body. Figure 15 shows that Case $1 \mathrm{H}$ provides maximum convective heat transfer because the blockage effect of the solid body is overwhelmed with tortuous effect of the porous layer. Location $2 \mathrm{H}$ shows the minimum convective heat transfer, referring to the destroyed momentum exchange across the porous-fluid interface. However, there is no significant effect of the body location when $\mathrm{Ra} \leq 10^{4}$,

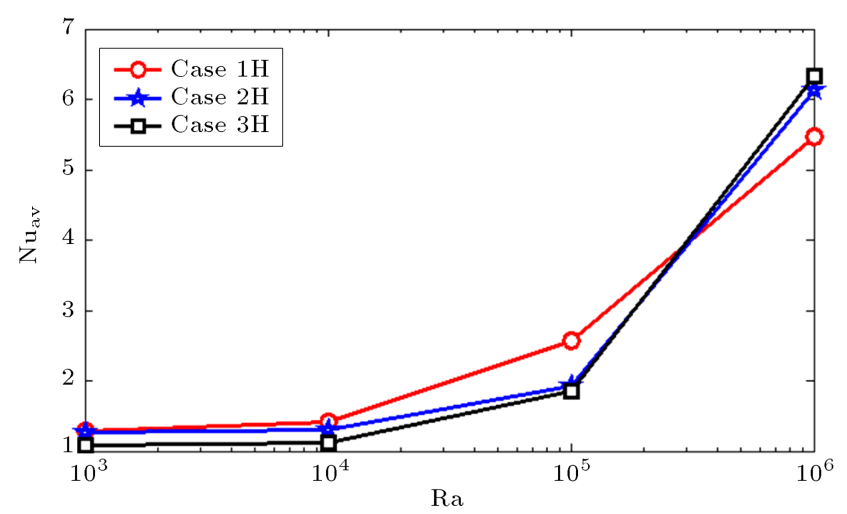

Figure 15. Effects of the horizontal body position on the average Nusselt number for $\mathrm{Da}=10^{-3}$, Le $=10, N=1$, $A=0.3$, and $\mathrm{Kr}=5$.

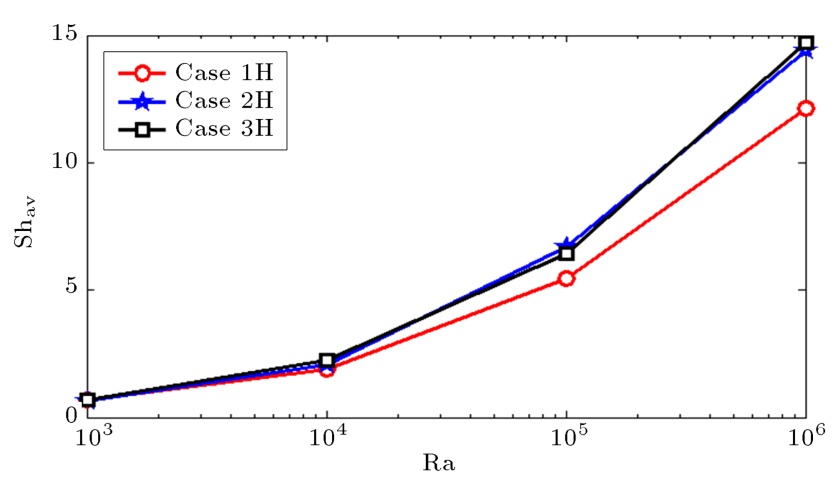

Figure 16. Effects of the horizontal body position on the average Sherwood number for $\mathrm{Da}=10^{-3}$, Le $=10$, $N=1, A=0.3$, and $\mathrm{Kr}=5$.

while locating the body in either porous or fluid layer $(1 \mathrm{H}$ or $3 \mathrm{H})$ can affect Nusselt number when Ra $>10^{5}$. As stated above, location $1 \mathrm{H}$ experiences a significant disturbance to the solutal boundary layer close to the species source. As such, the average Sherwood number is minimum due to this location. Nevertheless, Sherwood number looks unaffected by the other two positions, $2 \mathrm{H}$ and $3 \mathrm{H}$.

\subsubsection{Various vertical body locations}

The vertical body locations were chosen as follows:

- Case 1V: $Y_{o}=0$ corresponds to the lower position close to the adiabatic lower wall;

- Case 2V: $Y_{o}=0.35$ is in the cavity center, which is exactly similar to case $2 \mathrm{H}$;

- Case 3V: $Y_{0}=0.7$ addresses the top body position close to the top adiabatic wall.

The body in all these three cases is centered along the interface line. Case $2 \mathrm{~V}$ is similar to Case $2 \mathrm{H}$, discussed in the previous section. However, Cases $1 \mathrm{~V}$ and $3 \mathrm{~V}$ experience stronger streamlines. In Case $1 \mathrm{~V}$, the fluid circulates in a single-eye $\mathrm{CW}$ vortex with a core free of solid body. This core is localized in the upper part of the cavity, leaving a stagnant zone in the right lower corner as shown in Figure 19(a). For Case 3V, the space available brings the core to the lower part of the cavity. It is worth mentioning that when the fluid falls from the upper part of the cavity, it will be improved by the gravity action. Therefore, the strongest streamlines are seen in this case. Table 2 demonstrates the values of the minimum stream functions of the three vertical cases for different Rayleigh numbers.

The middle column of Figure 17 shows that the horizontal pattern of the isotherms deforms greatly at Case 2V. On the other hand, the convection fashion is reserved with the other two positions, $1 \mathrm{~V}$ and $3 \mathrm{~V}$. The solutal boundary layers presented in the right column of Figure 17 depict less effect with the vertical body positions. 


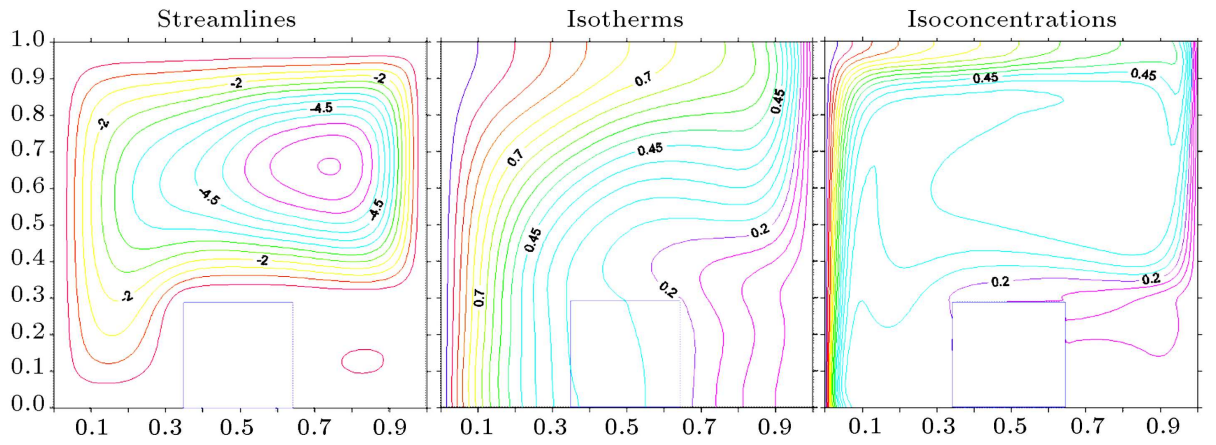

(a) Case $1 \mathrm{~V}$

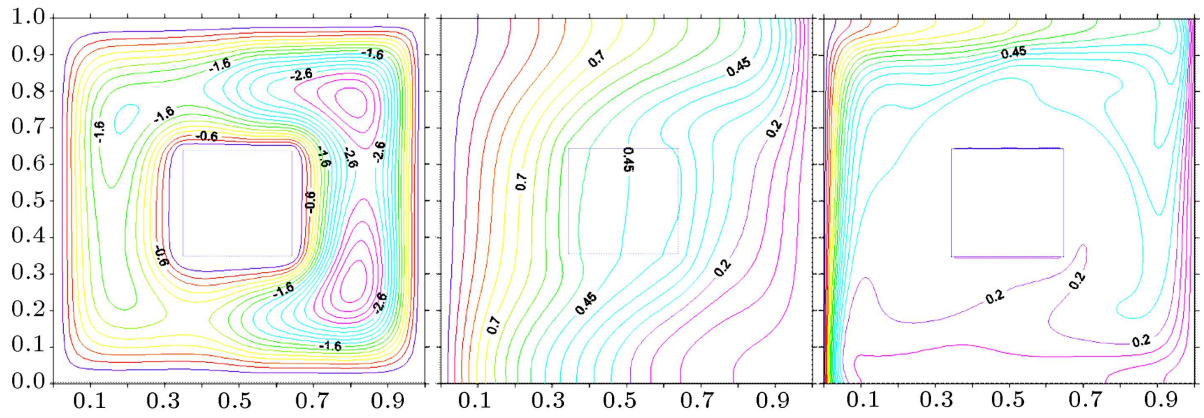

(b) Case $2 \mathrm{~V}$

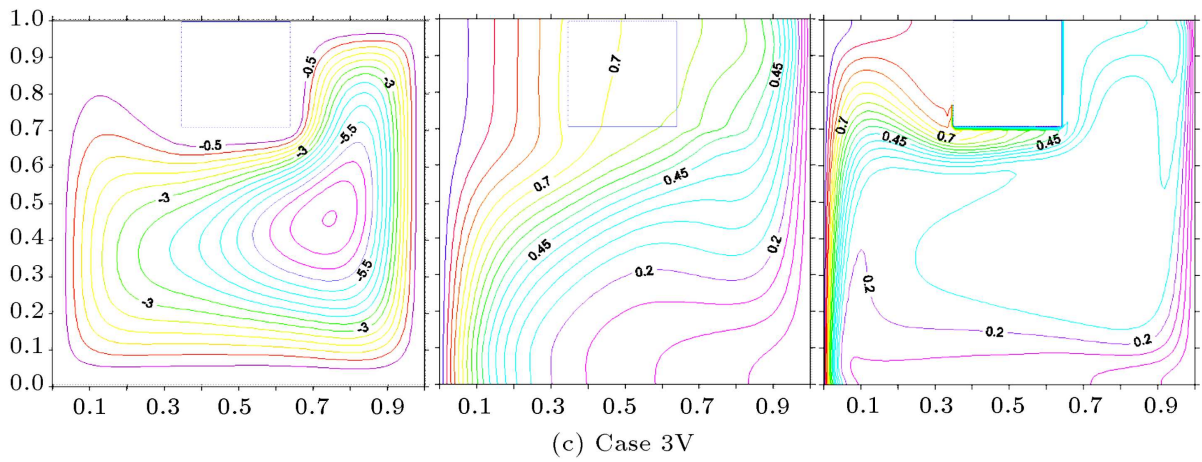

Figure 17. Effects of the vertical body position on the streamlines, isotherms, and isoconcentrations contours for Da $=$ $10^{-3}, \mathrm{Le}=10, N=1, A=0.3, \mathrm{Kr}=5$, and $\mathrm{Ra}=10^{5}$.

The average Nusselt number shown in Figure 18 shows that Case $2 \mathrm{~V}$ is the worst from the convection point of view. In this respect, the weakest fluid circulation in this case experiences lesser momentum

Table 2. Minimum stream functions' values as a function of the horizontal body position and Rayleigh number for $\mathrm{Da}=10^{-3}, \mathrm{Le}=10$, and $\mathrm{Kr}=5$.

\begin{tabular}{cccc} 
Ra & Case 1V & Case 2V & Case 3V \\
\hline $10^{3}$ & $-2.74 \mathrm{E}-01$ & $-1.21 \mathrm{E}-01$ & $-2.84 \mathrm{E}-01$ \\
$10^{4}$ & -1.72489 & -0.7805 & -1.83129 \\
$10^{5}$ & -6.53615 & -3.53763 & -7.52761 \\
$10^{6}$ & -15.3385 & -13.1729 & -19.3112 \\
\hline
\end{tabular}

exchange across the interface. $1 \mathrm{~V}$ and $3 \mathrm{~V}$ have the same convective heat transfer for Rayleigh number range of $\mathrm{Ra} \leq 10^{5}$.

The variations of Sherwood number shown in Figure 19 demonstrate that the mass transfer within the cavity is not affected by the body vertical location in the moderate and high Rayleigh numbers. At lower Rayleigh numbers, the flow circulation is already weak; hence, the worst position $2 \mathrm{~V}$ can bring about and add extra weakness to the streamlines. This in turn leads to less number of species carried by the convective effect; then, the Sherwood number decreases.

\section{Conclusions}

An in-house FORTRAN code was used in the present paper to study the double diffusive natural convection inside a halved-layered porous cavity with an inner thermally conductive square body. The study was 


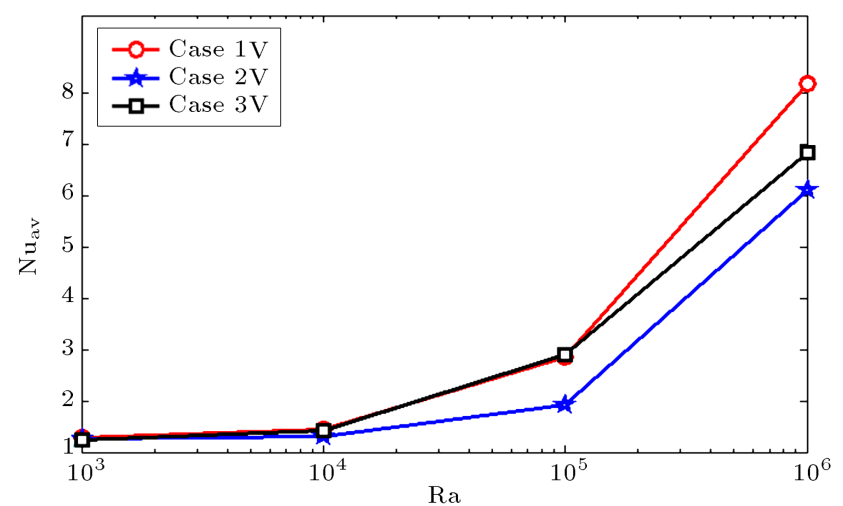

Figure 18. Effects of the vertical body position on the average Nusselt number for $\mathrm{Da}=10^{-3}$, Le $=10, N=1$, $A=0.3$, and $\mathrm{Kr}=5$.

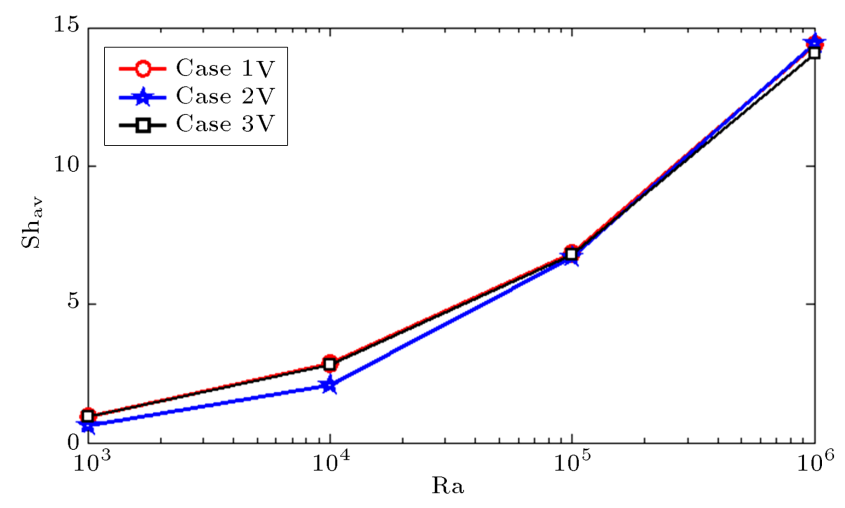

Figure 19. Effects of the vertical position on the average Sherwood number for $\mathrm{Da}=10^{-3}$, Le $=10, N=1$, $A=0.3$, and $\mathrm{Kr}=5$.

achieved numerically using the finite difference method with the upwind scheme procedure. The results have led to the following conclusions:

1. Both the mass transfer and convective heat transfer have a rapid increase when the Darcy number increases within the range of $\mathrm{Da}=10^{-6}-10^{-2}$;

2. The mass transfer is a continuously increasing function of Lewis number, while the convective heat transfer decreases with Lewis number;

3. The convective heat transfer is an increasing function of Rayleigh number; as a result, the mass transfer follows this fashion;

4. Both mass transfer and convective heat transfer increase with the increasing thermal conductivity of the solid square body. Nevertheless, the increment of mass transfer becomes limited at high Rayleigh number;

5. The best position of the solid body that gives maximum Nusselt number is close to the mid height of the left hot wall, while the worst position is at the cavity center;

6. On the contrary, the best position of the solid body that gives maximum Sherwood number is at the cavity center, while the worst position is close to the left high concentration wall.

\section{Nomenclature}

$A$

C

$D$

$\mathrm{Da}$

$g$

$H$

$h$

$k$

K

$\mathrm{Kr}$

$N$

$n$

$\mathrm{Nu}$

$\mathrm{Pr}$

$\mathrm{Ra}$

$\mathrm{Rd}$

$\mathrm{Sh}$

$T$

$u, v$

$U, V$

$W_{p}$

$x, y$

$X, Y$

Aspect ratio, $y_{b} / H$

Dimensionless concentration

Mass diffusivity $\left(\mathrm{m}^{2} \mathrm{~s}^{-1}\right)$

Darcy number

Gravitational acceleration $\left(\mathrm{m} \mathrm{s}^{-2}\right)$

Height of the cavity $(\mathrm{m})$

Local heat transfer coefficient (W $\mathrm{m}^{-2} \mathrm{~K}^{-1}$ )

Thermal conductivity ( $\mathrm{W} \mathrm{m}^{-1} \mathrm{~K}^{-1}$ )

Permeability of the porous medium $\left(\mathrm{m}^{2}\right)$

Conductivity ratio

Buoyancy ratio

Normal vector

Nusselt number

Prandtl number

Rayleigh number

Diffusivity ratio

Sherwood number

Temperature $(\mathrm{k})$

Velocity components $\left(\mathrm{ms}^{-1}\right)$

Dimensionless velocities components, $V=v H / \alpha, U=u H / \alpha$

Porous layer thickness $(\mathrm{m})$

Dimensionless coordinates $(\mathrm{m})$

Dimensionless coordinates, $X=x / H$, $Y=y / H$

\section{Greek symbols}

$\alpha$

$\beta$

$\varepsilon$

$\theta$

$\rho$

$\Psi$

$\Omega$

\section{Subscripts}

$\begin{array}{ll}\text { av } & \text { Average } \\ b & \text { Body } \\ c & \text { Cold } \\ \text { eff } & \text { Effective }\end{array}$

Thermal diffusivity $\left(\mathrm{m}^{2} \mathrm{~s}^{-1}\right)$

Thermal expansion coefficient $\left(K^{-1}\right)$

Porosity of the porous layer

Dimensionless temperature

Density $\left(\mathrm{kg} \mathrm{m}^{-3}\right)$

Dimensionless stream function

Dimensionless vorticity 


$\begin{array}{ll}f & \text { Fluid } \\ h & \text { Hot, high } \\ L & \text { Low } \\ p & \text { Porous }\end{array}$

\section{References}

1. Ingham, D.B. and Pop, I. (Eds.), Transport Phenomena in Porous Media II, 1st Ed., Pergamon (2002).

2. Baytas, A.C, Baytas, A.F., Ingham, D.B., and Pop, I. "Double diffusive natural convection in an enclosure filled with a step type porous layer: Non-Darcy flow" Int. J. Therm. Sci., 48, pp. 665-673 (2009).

3. Hirata, S.C., Goyeau, B., and Gobin, D. "Stability of thermosolutal natural convection in superposed fluid and porous layers", Transp. Porous Media, 78, pp. 525536 (2009).

4. Islam, A.W., Sharif, A.R., and Carlson, E.S. "Numerical investigation of double diffusive natural convection of $\mathrm{CO}_{2}$ in a brine saturated geothermal reservoir", Geothermics, 48, pp. 101-111 (2013).

5. Hadidi, N., Bennacer, R., and Ould-amer, Y. "Twodimensional thermo-solutal natural convective heat and mass transfer in a bi-layered and inclined porous enclosure", Energy, 93, pp. 2582-2592 (2015).

6. Mharzi, M., Daguenet, M., and Daoudi, S. "Thermosolutal natural convection in a vertically layered fluidporous medium heated from the side", Energy Conversion and Management, 41, pp. 1065-1090 (2000).

7. Bennacer, R., Beji, H., and Mohamad, A.A. "Double diffusive convection in a vertical enclosure inserted with two saturated porous layers confining a fluid layer", Int. J. Therm. Sci., 42, pp. 141-151 ( 2003).

8. Gobin, D., Goyeau, B., and Neculae, A. "Convective heat and solute transfer in partially porous cavities", Int. J. Heat Mass Transfer, 48, pp. 1898-1908 (2005).

9. Outaleb, Y., Bouhadef, K., and Rahli, O. "Double diffusive convection in a partially porous cavity under suction/injection effects", World Academy of Science, Engineering and Technology, 55, pp. 883-887 (2011).

10. Hadidi, N, Ould-Amer, Y., and Bennacer, R. "Bilayered and inclined porous collector: Optimum heat and mass transfer", Energy, 51, pp. 422-430 (2013).

11. Hadidi, N. and Bennacer, V. "Three-dimensional double diffusive natural convection across a cubical enclosure partially filled by vertical porous layer", Int. J. Therm. Sci., 101, pp. 143-157 (2016).

12. House, J.M., Beckermann, C., and Smith, T.F. "Effect of a centered conducting body on natural convection heat transfer in an enclosure", Numer. Heat Transfer, A, 18, pp. 213-225 (1990).

13. Ha, M.Y. and Jung, M.J. "A numerical study on three-dimensional conjugate heat transfer of natural convection and conduction in a differentially heated cubic enclosure with a heat-generating cubic conducting body", Int. J. Heat Mass Transfer, 43, pp. 4229-4248 (2000).
14. Lee, J.R. and Ha, M.Y. "A numerical study of natural convection in a horizontal enclosure with a conducting body", Int. J. Heat Mass Transfer, 48, pp. 3308-3318 (2005).

15. Braga, E.J. and de Lemos, M.J.S. "Heat transfer in enclosures having a fixed amount of solid material simulated with heterogeneous and homogeneous models", Int. J. Heat Mass Transfer, 48, pp. 4748-4765 (2005).

16. Braga, E.J. and de Lemos, M.J.S. "Laminar natural convection in cavities filled with circular and square rods", Int. Commun. Heat Mass Transfer, 32, pp. 1289-1297 (2005).

17. Merrikh, A.A. and Lage, J.L. "Natural convection in an enclosure with disconnected and conducting solid blocks", Int. J. Heat Mass Transfer, 48, pp. 1361-1372 (2005).

18. Das, M.K. and Reddy, K.S.K. "Conjugate natural convection heat transfer in an inclined square cavity containing a conducting block", Int. J. Heat Mass Transfer, 49, pp. 4987-5000 (2006).

19. Zhao, F., Liu, D., and Tang, G. "Conjugate heat transfer in square enclosure", Heat Mass Transfer, 43, pp. 907-922 (2007).

20. Sheremet, M.A., Oztop, H.F., Pop, I., and AbuHamdeh, N. "Analysis of entropy generation in natural convection of nanofluid inside a square cavity having hot solid block: Tiwari and Das' Model", Entropy, 18, pp. 1-15 (2016).

21. Sivaraj, C. and Sheremet, M.A. "MHD natural convection in an inclined square porous cavity with a heat conducting solid block", Journal of Magnetism and Magnetic Materials, 426, pp. 351-360 (2017).

22. Chamkha, A.J., Selimefendigil, F., and Ismael, M.A. "Mixed convection in a partially layered porous cavity with an inner rotating cylinder", Numer. Heat Transfer, A, 69, pp. 659-675 (2016).

23. Selimefendigil, F., Ismael, M.A., and Chamkha, A.J. "Mixed convection in superposed nanofluid and porous layers in square enclosure with inner rotating cylinder", Int. Journal of Mechanical Sciences, 124(125), pp. 95-108,(2017).

24. Chamkha, A.J. and Ismael, M.A. "Natural convection in differentially heated partially porous layered cavities filled with nanofluid", Numer. Heat Transfer, A, 65, pp. 1089-1113 (2014).

25. Sheremet, M.A. and Trifonova, T.A. "Unsteady conjugate natural convection in a vertical cylinder containing a horizontal porous layer: Darcy model and brinkman extended Darcy model", Transp. Porous Med., 101, pp. 437-463 (2014).

26. M-Juan, Y., B-Ming, Y., Bin, Z., and M-Tao, H.A. "Geometry model for tortuosity of streamtubes in porous media with spherical particles", Chines Physics Letters, 22, pp. 1464-1467 (2005). 
27. Holman, J.P., Heat Transfer, McGraw Hill, New York, 10th Ed. (2010).

\section{Biographies}

Muneer AbdulJaleel Ismael received BSc, MSc, and $\mathrm{PhD}$ degrees from University of Basrah, Basrah, Iraq in 1996, 1998, and 2007, respectively. $\mathrm{He}$ is currently an Assistance Professor in the Mechanical Engineering Department at the University of Basrah, Iraq. In his postgraduate projects, he worked on designing the electro-magnetic flowmeter for partially filled pipes. His research interests included numerical modeling, porous media, convective heat transfer, nanofluid, and flow measurement.

Hassan Saad Ghalib received BSc and MSc degrees from University of Basrah, Basrah, Iraq in 2014 and 2017, respectively. He is currently working in Bonatti Oil Company. His research interests included heat and mass transfer, porous media, and numerical modeling. 